\title{
An optimal strongly identifying code in the infinite triangular grid
}

\author{
Iiro Honkala* \\ Department of Mathematics \\ University of Turku \\ 20014 Turku, Finland \\ honkala@utu.fi
}

Submitted: Aug 18, 2009; Accepted: Jun 15, 2010; Published: Jun 29, 2010

Mathematics Subject Classification: 05C69, 68R10

\begin{abstract}
Assume that $G=(V, E)$ is an undirected graph, and $C \subseteq V$. For every $\mathbf{v} \in V$, we denote by $I(\mathbf{v})$ the set of all elements of $C$ that are within distance one from $\mathbf{v}$. If the sets $I(\mathbf{v}) \backslash\{\mathbf{v}\}$ for $\mathbf{v} \in V$ are all nonempty, and, moreover, the sets $\{I(\mathbf{v}), I(\mathbf{v}) \backslash\{\mathbf{v}\}\}$ for $\mathbf{v} \in V$ are disjoint, then $C$ is called a strongly identifying code. The smallest possible density of a strongly identifying code in the infinite triangular grid is shown to be $6 / 19$.
\end{abstract}

Keywords: Graph, identifying code, triangular grid, density.

\section{Introduction}

Assume that $G=(V, E)$ is an undirected graph with vertex set $V$ and edge set $E$. A subset $C \subseteq V$ is called a code in $G$, and its elements are called codewords.

The distance $d(\mathbf{u}, \mathbf{v})$ between two vertices $\mathbf{u}$ and $\mathbf{v}$ is the number of edges on any shortest path between them.

For all $\mathbf{v} \in V$ we denote

$$
I(\mathbf{v})=\{\mathbf{c} \in C: d(\mathbf{c}, \mathbf{v}) \leqslant 1\} .
$$

If we denote by $B_{r}(\mathbf{v})$ the ball of radius $r$ with centre $\mathbf{v}$, then $I(\mathbf{v})=C \cap B_{1}(\mathbf{v})$.

If all the sets $I(\mathbf{v})$ are nonempty and pairwise different, then $C$ is called an identifying code. This concept was introduced in [8] in connection with studying multiprocessor

*Research supported by the Academy of Finland under grant 210280. 
architectures. Such an architecture can be viewed as a graph, where each vertex represents a processor, and each edge represents a dedicated link between two processors. Assume that at most one of the processors is malfunctioning. Each of the chosen codewords $\mathbf{c}$ tests the sets $B_{1}(\mathbf{c})$ and reports YES if it detects a problem and NO otherwise. The fact that $C$ is identifying implies that based on the reports, we can uniquely identify the one malfunctioning processor or tell that everything is fine.

Strongly identifying codes were introduced in [7] (in a more general form); cf. also [14].

Definition 1. A code $C$ in the graph $G=(V, E)$ is called strongly identifying if all the sets $I(\mathbf{v}) \backslash\{\mathbf{v}\}$ for $\mathbf{v} \in V$ are nonempty, and, moreover, the sets $\{I(\mathbf{v}), I(\mathbf{v}) \backslash\{\mathbf{v}\}\}$ for $\mathbf{v} \in V$ are disjoint.

Here the idea is that a malfunctioning processor may or may not be able to send a correct report, and we need a slightly stronger code. Clearly, a strongly identifying code is also identifying.

Strongly identifying codes have also been studied in [9] and [10].

The concept of a locating-dominating set introduced by Slater [13] (see also [3]) is closely related to that of identifying codes.

Identifying codes and locating-dominating codes have been extensively studied: see the Internet bibliography [11] maintained by Antoine Lobstein. For results on the triangular grid, see, e.g., [1], [2], [4], [6] and [5].

In the square grid $\mathbb{Z}^{2}$ it is easy to see that the smallest possible density of a strongly identifying code equals $2 / 5$. Indeed, the code $\left\{(x, y) \in \mathbb{Z}^{2}: x \equiv 1\right.$ or $\left.3(\bmod 5)\right\}$ is strongly identifying and has density $2 / 5$. The lower bound $2 / 5$ on the density is an immediate corollary of [12, Theorem 14].

In the hexagonal mesh the smallest possible density of a strongly identifying code equals $1 / 2$. Indeed, if we delete all the vertical edges in the hexagonal mesh, and take as codewords all the vertices on every second of the resulting (infinitely many) doubly infinite paths, we clearly get a strongly identifying code with density $1 / 2$. The lower bound on the density is an immediate corollary of [12, Theorem 14].

From now on we consider the infinite triangular grid. The vertex set of the infinite triangular grid $T$ is $V=\{v(i, j): i, j \in \mathbb{Z}\}$, where

$$
v(i, j)=i(1,0)+j\left(\frac{1}{2}, \frac{\sqrt{3}}{2}\right),
$$

and two vertices are adjacent if their Euclidean distance is 1 . Denote by $T_{n}$ the set of vertices $v(i, j)$ with $|i| \leqslant n$ and $|j| \leqslant n$. The density of a code $C$ in $T$ is defined to be

$$
D(C)=\limsup _{n \rightarrow \infty} \frac{\left|C \cap T_{n}\right|}{\left|T_{n}\right|} .
$$

The smallest density of an identifying code in the infinite triangular grid is $1 / 4$; see [8].

It is easy to check that the code given in Figure 1 is strongly identifying and has density of 6/19. We always denote codewords by black dots. The code is obtained as a doubly periodic tiling and the tile is shown in the figure. 


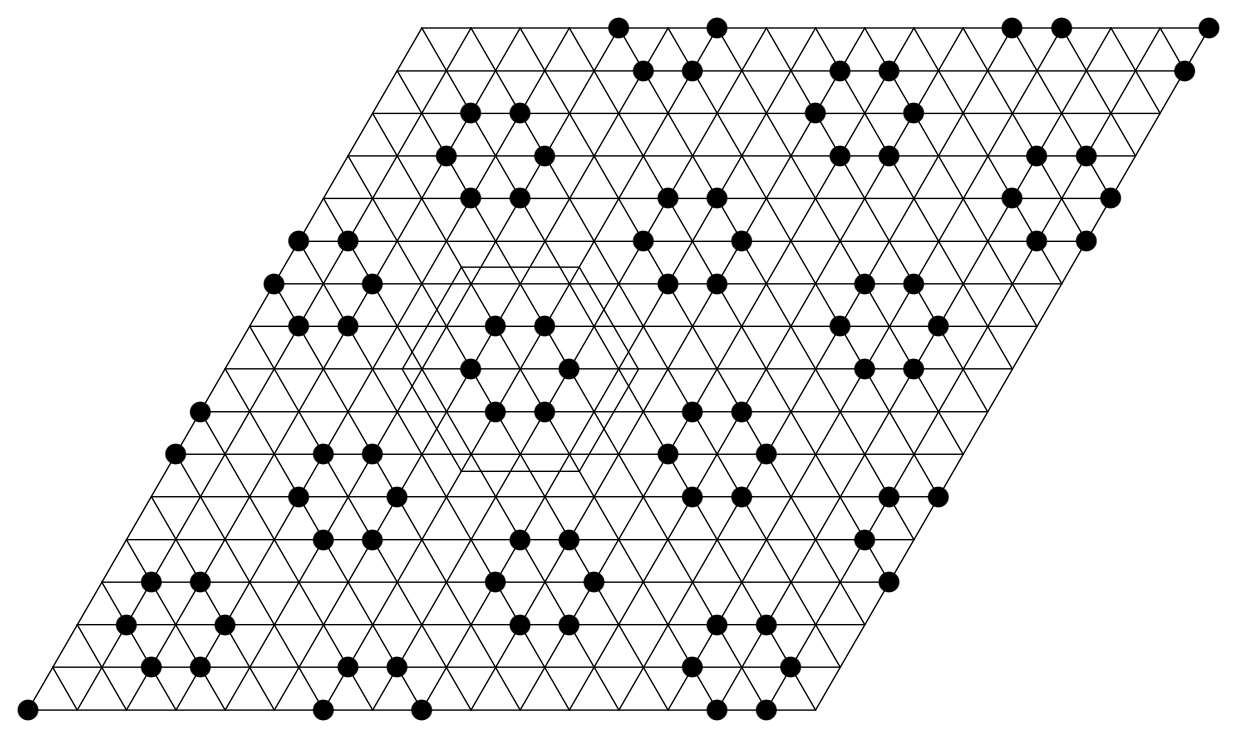

Figure 1: A strongly identifying code with density 6/19.

The purpose of this note is to prove that this code is optimal, i.e., the density of every strongly identifying code in the infinite triangular grid is at least 6/19.

\section{The proof}

From now on we assume that $C$ is a strongly identifying code in $T$.

Denote

$$
C_{i}=\{\mathbf{c} \in C:|I(\mathbf{c})|=i\}
$$

and

$$
C_{\geqslant j}=\bigcup_{i \geqslant j} C_{i}
$$

We also denote

$$
N_{i}=\{\mathbf{v} \notin C:|I(\mathbf{v})|=i\}
$$

and

$$
N_{\geqslant j}=\bigcup_{i \geqslant j} N_{i}
$$

Trivially, $C_{0}=C_{1}=\emptyset$.

Following Slater [14] we define the share of a codeword $\mathbf{c} \in C$ - which we denote by $s(\mathbf{c})$ - by the formula

$$
s(\mathbf{c})=\sum_{\mathbf{v} \in B_{1}(\mathbf{c})} \frac{1}{|I(\mathbf{v})|} .
$$

We now introduce a voting scheme using which we perform an averaging over the shares of the codewords. 


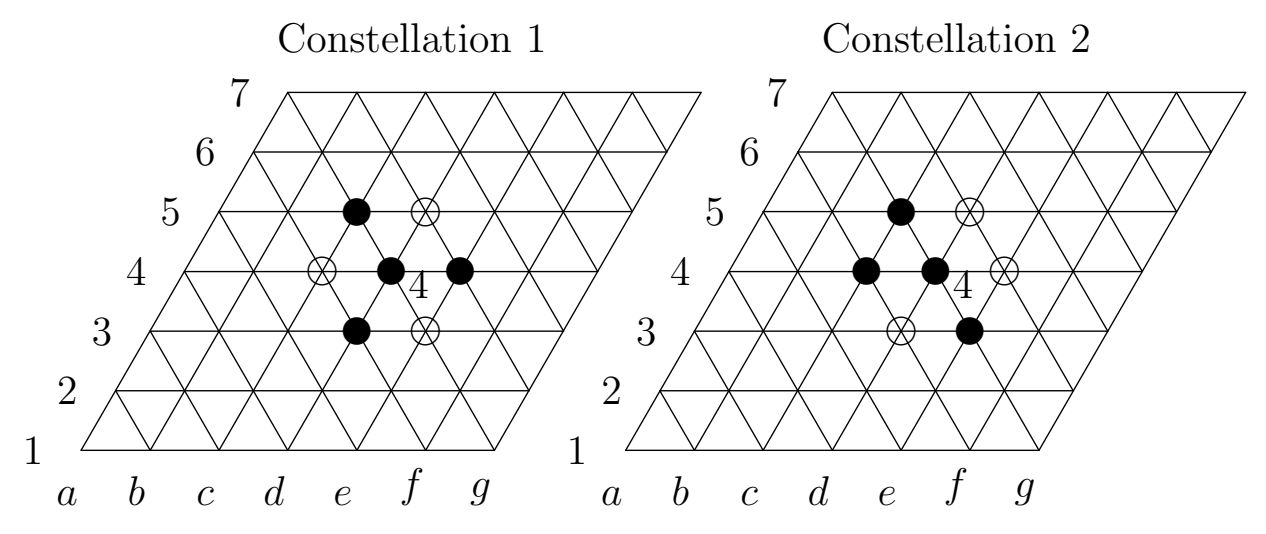

Figure 2: Constellations 1 and 2.

Rule 1: Every codeword in $C_{\geqslant 4}$ gives $1 / 3$ of a vote to every codeword neighbour in $C_{2}$.

In fact, no codeword can have more than one codeword neighbour that belongs to $C_{2}$ : if $\mathbf{a} \in C_{2}$ and $\mathbf{b} \in C_{2}$ were two different codeword neighbours of $\mathbf{c} \in C$, then

$$
I(\mathbf{a}) \backslash\{\mathbf{a}\}=\{\mathbf{c}\}=I(\mathbf{b}) \backslash\{\mathbf{b}\}
$$

and the code would not be strongly identifying.

Rule 2: Every codeword $\mathbf{c} \in C_{\geqslant 4}$ gives $1 / 12$ of a vote to every codeword neighbour in $C_{3}$ and all their codeword neighbours in $C_{3}$ (i.e., if $S$ is the subset of codewords of $C_{3}$ referred to above, then each element of $S$ gets $1 / 12$ of a vote from $\mathbf{c}$ ).

Clearly, if $\mathbf{c} \in C_{\geqslant 4}$ and $\mathbf{a} \in C_{3}$ is its codeword neighbour, then a can have at most one codeword neighbour in $C_{3}$.

These two rules describe the voting behaviour of the vertices in $C_{\geqslant 4}$.

Lemma 1. If $\mathbf{c} \in C_{\geqslant 4}$, then $s(\mathbf{c}) \leqslant 19 / 6$ and $\mathbf{c}$ gives at most $19 / 6-s(\mathbf{c})$ votes.

Proof. Consider first the vertices $\mathbf{c} \in C_{4}$. There are essentially three different cases.

Assume first that none of the codeword neighbours of $\mathbf{c}$ are adjacent. Without loss of generality, $\mathbf{c}$ is the vertex d4 of Constellation 1 in Figure 2. As we already remarked after introducing Rule 1, at most one of the codewords c5, d3 and e4 is in $C_{2}$, and the others are in $C_{\geqslant 3}$. The vertices c $4, \mathrm{~d} 5$ and $\mathrm{e} 3$ are all in $N_{\geqslant 3}$, and therefore

$$
s(\mathbf{c}) \leqslant \frac{1}{4}+5 \cdot \frac{1}{3}+\frac{1}{2}=\frac{29}{12}
$$

and $19 / 6-s(\mathbf{c}) \geqslant 9 / 12$. The number of votes given by $\mathbf{c}$ is clearly at most $1 / 3+4 \cdot 1 / 12=$ $8 / 12 \leqslant 9 / 12$ as claimed. 


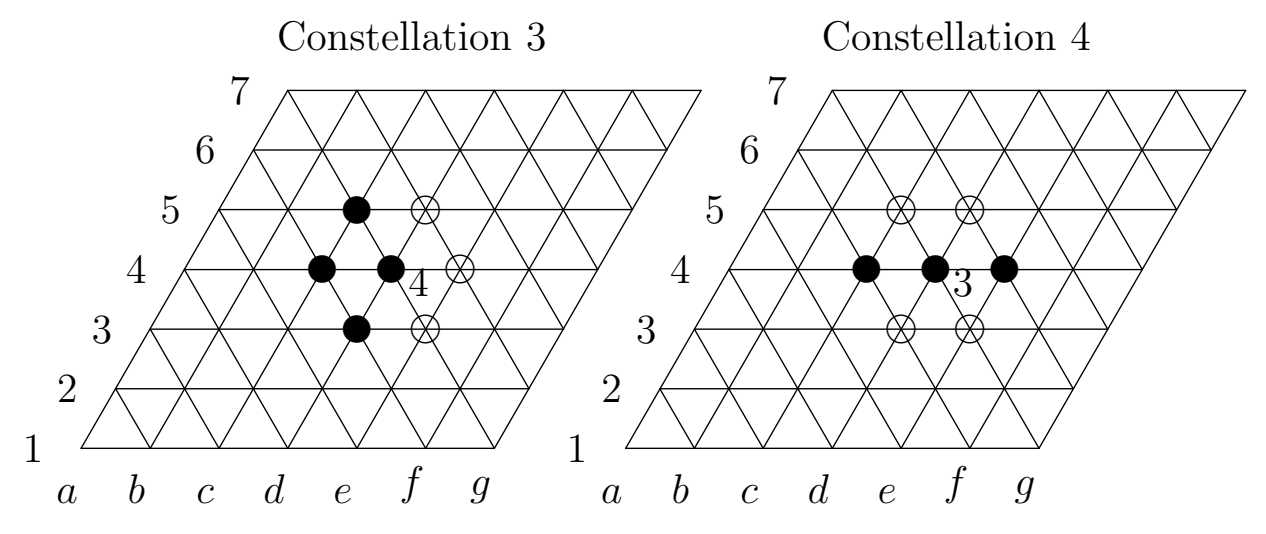

Figure 3: Constellations 3 and 4.

Assume then that $\mathbf{c}$ is like $\mathrm{d} 4$ in Constellation 2 in Figure 2. Then $\mathrm{c} 4, \mathrm{c} 5 \in C_{\geqslant 3}$, and at least one of the vertices $\mathrm{c} 4$ and $\mathrm{c} 5$ is in $C_{\geqslant 4}$ (otherwise $I(\mathrm{c} 4)=I(\mathrm{c} 5)=\{\mathrm{c} 4, \mathrm{c} 5, \mathrm{~d} 4\}$ ). If e3 $\in C_{2}$, then e4 $\in N_{\geqslant 3}$ (otherwise $I(\mathrm{e} 3)=I(\mathrm{e} 4)=\{\mathrm{e} 3, \mathrm{~d} 4\}$ ); and for the same reason if e $4 \in N_{2}$, then e $3 \in C_{\geqslant 3}$. Anyway,

$$
s(\mathbf{c}) \leqslant 2 \cdot \frac{1}{4}+3 \cdot \frac{1}{3}+2 \cdot \frac{1}{2}=\frac{5}{2},
$$

and $19 / 6-s(\mathbf{c}) \geqslant 2 / 3$. The number of votes given by $\mathbf{c}$ is clearly at most $2 / 3$.

Assume then that $\mathbf{c}$ is like the vertex $\mathrm{d} 4$ in Constellation 3 in Figure 3. It is possible that e4 $\in N_{1}$. However, at least one of the codewords d3 and c5 belongs to $C_{\geqslant 4}$ : if both of them were in $C_{3}$, then $I(\mathrm{~d} 3) \backslash\{\mathrm{d} 3\}=I(\mathrm{c} 5) \backslash\{\mathrm{c} 5\}$, contradicting the fact that $C$ is a strongly identifying code. This implies that

$$
s(\mathbf{c}) \leqslant 3 \cdot \frac{1}{4}+\frac{1}{3}+2 \cdot \frac{1}{2}+1=\frac{37}{12}
$$

and $19 / 6-s(\mathbf{c}) \geqslant 1 / 12$, but it also implies that $\mathbf{c}$ gives at most $1 / 12$ of a vote: if neither c5 nor d 3 is in $C_{3}$, then $\mathbf{c}$ gives no votes at all; if c5, say, is in $C_{3}$, then it gets $1 / 12$ of a vote from $\mathbf{c}$, but neither of the codeword neighbours of c5 is in $C_{3}$.

If $\mathbf{c} \in C_{\geqslant 5}$, then trivial calculations show that in all cases $s(\mathbf{c}) \leqslant 5 / 2$ and that $\mathbf{c}$ gives at most $2 / 3$ of a vote.

The final three voting rules tell how the vertices of $C_{3}$ vote.

Rule 3: If a codeword $\mathbf{c} \in C_{3}$ has a codeword neighbour $\mathbf{a} \in C_{2}$, then $\mathbf{c}$ gives $1 / 3$ of a vote to $\mathbf{a}$, if $\mathbf{c}$ and its two codeword neighbours are collinear, and $1 / 4$ of a vote, otherwise.

Rule 4: Assume that $\mathbf{c} \in C_{3}$ and that $\mathbf{c}$ has a codeword neighbour that belongs to $C_{2}$ or that $\mathbf{c}$ and its two codewords neighbours are collinear. Then c gives $1 / 12$ of a vote to every codeword neighbour in $C_{3}$ and all their codeword neighbours in $C_{3} \backslash\{\mathbf{c}\}$. 


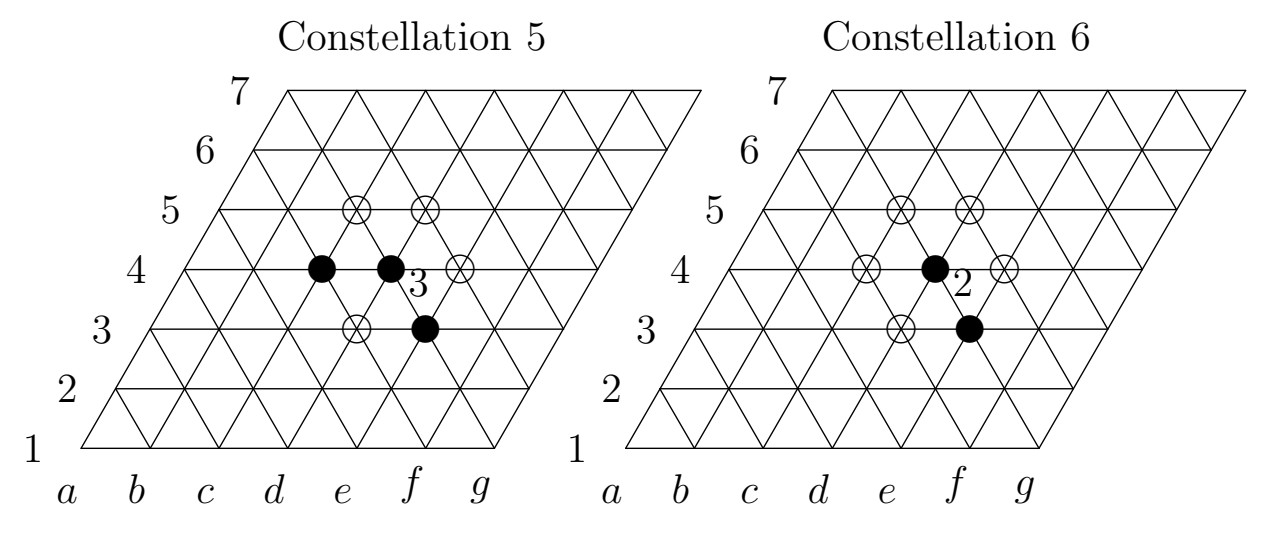

Figure 4: Constellations 5 and 6.

Rule 5: Assume that $\mathbf{c}$ and its two codeword neighbours all belong to $C_{3}$ but are not collinear. If the share of $\mathbf{c}$ is at most $37 / 12$ and the share of exactly one of its codeword neighbours is bigger than $19 / 6$, then that codeword gets $1 / 12$ of a vote from $\mathbf{c}$.

Lemma 2. Assume that $\mathbf{c} \in C_{3}$ and none of its neighbours belongs to $C_{2}$, and that $\mathbf{c}$ gives votes. Then $s(\mathbf{c}) \leqslant 19 / 6$ and $\mathbf{c}$ gives at most $19 / 6-s(\mathbf{c})$ votes.

Proof. The case when $\mathbf{c}$ gives votes according to Rule 5 is trivial; so assume that $\mathbf{c}$ does not give any votes according to Rule 5 . Then $\mathbf{c}$ gives votes according to Rule 4 , and hence $\mathbf{c}$ and its two codeword neighbours $\mathbf{a} \in C_{\geqslant 3}$ and $\mathbf{b} \in C_{\geqslant 3}$ are collinear. Without loss of generality, $\mathbf{c}$ is the vertex $\mathrm{d} 4$ in Constellation 4 in Figure 3 . Then at least one of the vertices $\mathrm{d} 3$ and c5 belongs to $N_{\geqslant 3}$; likewise at least one of the vertices e3 and d5 belongs to $N_{\geqslant 3}$. Consequently,

$$
s(\mathbf{c}) \leqslant 5 \cdot \frac{1}{3}+2 \cdot \frac{1}{2}=\frac{8}{3},
$$

and $19 / 6-s(\mathbf{c}) \geqslant 1 / 2$. According to Rule 4 , c gives $1 / 12$ of a vote to at most four vertices, and the claim is clear.

Lemma 3. Assume that $\mathbf{c} \in C_{3}$ has a neighbour $\mathbf{a} \in C_{2}$. Then $s(\mathbf{c}) \leqslant 19 / 6$ and $\mathbf{c}$ gives at most $19 / 6-s(\mathbf{c})$ votes.

Proof. Assume first that $\mathbf{c}$ and its two codeword neighbours are collinear. Without loss of generality, $\mathbf{c}$ is again the vertex $\mathrm{d} 4$ in Constellation 4 and $\mathrm{c} 4$ belongs to $C_{2}$ (and then obviously e4 is in $C_{\geqslant 3}$ ). Then both d3 and c5 belong to $N_{\geqslant 3}$; and at least one of the vertices e3 and d5 belongs to $N_{\geqslant 3}$. But again we see that $s(\mathbf{c}) \leqslant 8 / 3$, and $19 / 6-s(\mathbf{c}) \geqslant 1 / 2$, and the total number of votes given by $\mathbf{c}$ is at most $1 / 3+2 \cdot 1 / 12=1 / 2$.

Assume second that $\mathbf{c}$ and its two codeword neighbours are not collinear. Without loss of generality $\mathbf{c}$ is the vertex $\mathrm{d} 4$ in Constellation 5 in Figure 4 , and c4 belongs to $C_{2}$. Again, e3 is in $C_{\geqslant 3}$. Because $C$ is strongly identifying, we know that $I(\mathrm{c} 4) \backslash\{c 4\} \neq I(\mathrm{~d} 5)$, 


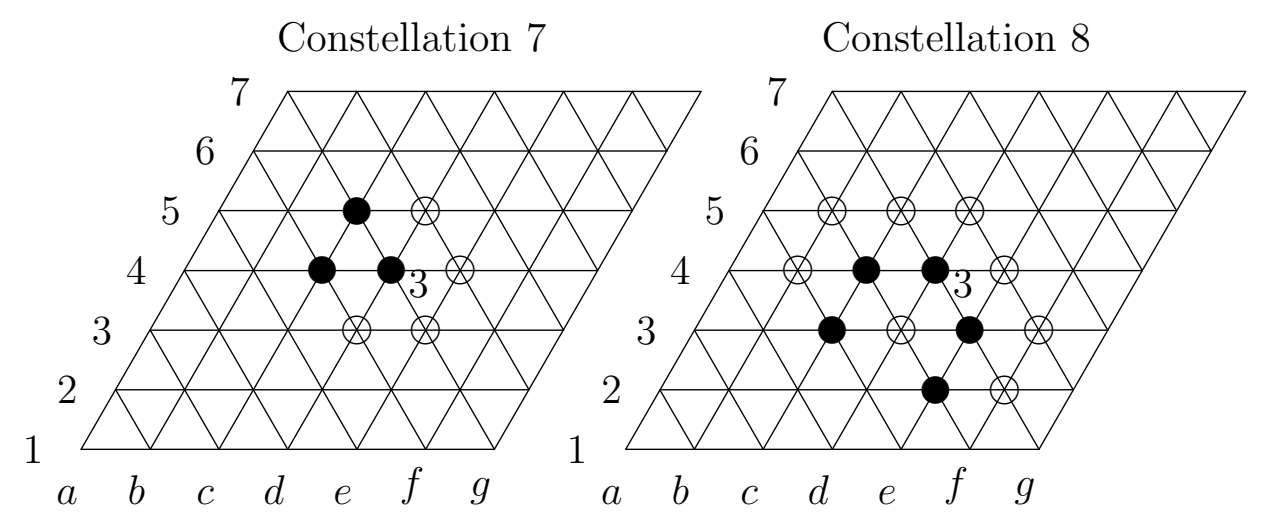

Figure 5: Constellations 7 and 8.

and therefore $\mathrm{d} 5 \in N_{\geqslant 2}$. Because $I(\mathrm{c} 5) \neq I(\mathrm{c} 4)$, we know that $\mathrm{c} 5 \in N_{\geqslant 3}$; and because $I(\mathrm{~d} 3) \neq I(\mathrm{~d} 4)$, we know that $\mathrm{d} 3 \in N_{\geqslant 4}$. All in all,

$$
s(\mathbf{c}) \leqslant \frac{1}{4}+3 \cdot \frac{1}{3}+3 \cdot \frac{1}{2}=\frac{11}{4},
$$

and $19 / 6-s(\mathbf{c}) \geqslant 5 / 12$. According to Rules 3 and 4 the vertex $\mathbf{c}$ gives $1 / 4$ of a vote to $\mathrm{c} 4$ and $1 / 12$ of a vote to at most two vertices, and hence at most 5/12 of a vote altogether.

Lemma 4. If $\mathbf{c} \in C_{2}$, then $\mathbf{c}$ gets at least $s(\mathbf{c})-19 / 6$ votes.

Proof. Without loss of generality, assume that $s(\mathbf{c})>19 / 6$ and that $\mathbf{c}$ is the vertex $\mathrm{d} 4 \mathrm{in}$ Constellation 6 in Figure 4. Because $C$ is identifying, we know that $I(\mathrm{~d} 3) \neq I(\mathrm{~d} 4)$ and hence d $3 \in N_{\geqslant 3}$; and similarly, e3 $\in C_{\geqslant 3}$ and e $4 \in N_{\geqslant 3}$. At most one of the vertices c4, c5 and d5 belongs to $N_{1}$ and at least two of them belong to $N_{\geqslant 2}$. Hence

$$
s(\mathbf{c}) \leqslant 3 \cdot \frac{1}{3}+3 \cdot \frac{1}{2}+1=\frac{7}{2} .
$$

Therefore, if e3 gives $1 / 3$ of a vote to $\mathrm{d} 4$, then the claim certainly holds. By Rules 1 and 3 this is true, unless e 3 belongs to $C_{3}$ and its remaining codeword neighbour is either e2 or f3. These are symmetrical cases, so assume that e 2 is in $C$ and $\mathrm{f} 2$ and $\mathrm{f} 3$ are noncodewords. Then $I(\mathrm{~d} 3) \neq I(\mathrm{e} 3)$ implies that $\mathrm{d} 3$ is in $N_{\geqslant 4}$. Hence $s(\mathbf{c}) \leqslant 41 / 12$, and $s(\mathbf{c})-19 / 6 \leqslant 1 / 4$, and the claim is again valid, because e 3 now gives $1 / 4$ of a vote to $\mathrm{d} 4$ by Rule 3 .

Lemma 5. If $\mathbf{c} \in C_{3}$ and $s(\mathbf{c})>19 / 6$, then $\mathbf{c}$ gets at least $s(\mathbf{c})-19 / 6$ votes.

Proof. Because $\mathbf{c} \in C_{3}$ and $s(\mathbf{c})>19 / 6$, we know that $\mathbf{c}$ and its two codeword neighbours cannot be collinear, and without loss of generality $\mathbf{c}$ is the vertex $\mathrm{d} 4$ in Constellation 5 or in Constellation 7. 
Consider first Constellation 7. Here, $\mathrm{c} 4$ and $\mathrm{c} 5$ both belong to $C_{\geqslant 4}$ and at most one of the vertices e3 and e4 can belong to $N_{1}$. Hence $s(\mathbf{c}) \leqslant 10 / 3$, and it suffices to show that $\mathrm{d} 4$ gets at least $1 / 6$ of a vote. But, indeed, both $\mathrm{c} 4$ and $\mathrm{c} 5$ give 1/12 of a vote to $\mathrm{d} 4$ by Rule 2.

Assume therefore that $\mathbf{c}$ is the vertex d4 in Constellation 5. The fact that $s(\mathbf{c})>19 / 6$ implies that d5 belongs to $N_{1}$. Because $I(\mathrm{c} 4) \backslash\{c 4\} \neq I(\mathrm{~d} 5)$, we know that $\mathrm{c} 4 \in C_{\geqslant 3}$ and similarly e3 $\in C_{\geqslant 3}$. Because $I(\mathrm{~d} 3) \neq I(\mathrm{~d} 4)$, we know that $\mathrm{d} 3 \in N_{\geqslant 4}$. Therefore

$$
s(\mathbf{c}) \leqslant \frac{1}{4}+3 \cdot \frac{1}{3}+2 \cdot \frac{1}{2}+1=\frac{13}{4} .
$$

The fact that $s(\mathbf{c})>19 / 6$ now implies that c5 and e4 are both in $N_{2}$. Therefore b5 and f3 are both non-codewords. The claim is clearly true if $\mathrm{d} 4$ gets at least $1 / 12$ of a vote. If c4 or e3 is in $C_{\geqslant 4}$, then this is true by Rule 2 , so we can assume that they both belong to $C_{3}$. If b4 is in $C$, then $\mathrm{c} 4$ gives $1 / 12$ of a vote to $\mathrm{d} 4$ by Rule 4 , so we can assume that b4 is not in $C$; similarly, we can assume that $\mathrm{f} 2$ is not in $C$. But then $\mathrm{c} 3$ and $\mathrm{e} 2$ are both in $C$ and we have Constellation 8 in Figure 5.

If $\mathrm{c} 3$ is in $C_{2}$, then c4 gives $1 / 12$ of a vote to $\mathrm{d} 4$ by Rule 4 ; so assume that $\mathrm{c} 3$ is in $C_{\geqslant 3}$. If c3 is in $C_{\geqslant 4}$, then c3 gives $1 / 12$ of a vote to d4 by Rule 2 ; so assume that c3 is in $C_{3}$.

If b3 is in $C$, then $s(\mathrm{c} 3)<19 / 6$ and $s(\mathrm{c} 4) \leqslant 37 / 12$ and therefore c4 gives $1 / 12$ of a vote to d4 by Rule 5 ; so assume that b3 is not in $C$.

Because $s(\mathbf{c})>19 / 6$, we know that $\mathrm{d} 2$ is not in $C$. But then $\mathrm{c} 2$ must be in $C$ and $\mathrm{c} 3$ gives $1 / 12$ of a vote to $\mathrm{d} 4$ by Rule 4 .

Theorem 1. The density of a strongly identifying code in the infinite triangular grid is at least 6/19.

Proof. Assume that $C$ is a strongly identifying code in the infinite triangular grid. Let $n \geqslant 5$ be fixed, and consider the set $T_{n}$.

Consider now the voting process described above. For all codewords c, define $m(\mathbf{c})$ as the total number of votes given minus the total number of votes received.

We first check that for all $\mathbf{c} \in C$ we have

$$
s(\mathbf{c})+m(\mathbf{c}) \leqslant \frac{19}{6} .
$$

There are several cases to consider:

- If $\mathbf{c} \in C_{\geqslant 4}$, then $\mathbf{c}$ gives at most $19 / 6-s(\mathbf{c})$ votes by Lemma 1 , and the sum of $s(\mathbf{c})$ and the number of votes given by $\mathbf{c}$ is at most $19 / 6$.

- If $\mathbf{c} \in C_{2}$, then $\mathbf{c}$ does not give any votes and by Lemma 4 , the number of votes received by $\mathbf{c}$ is at least $s(\mathbf{c})-19 / 6$, and again (1) holds.

- If $\mathbf{c} \in C_{3}$ gives votes, then by Lemmas 2 and 3 , $\mathbf{c}$ gives at most $19 / 6-s(\mathbf{c})$ votes, and (1) holds. 
- Finally, assume that $\mathbf{c} \in C_{3}$ does not give any votes. If $s(\mathbf{c}) \leqslant 19 / 6$, then (1) trivially holds. If $s(\mathbf{c})>19 / 6$, then by Lemma 5 , $\mathbf{c}$ gets at least $s(\mathbf{c})-19 / 6$ votes and again (1) holds.

Consider the sum

$$
\sum_{\mathbf{c} \in C \cap T_{n}}(s(\mathbf{c})+m(\mathbf{c})) .
$$

Except for the votes received from codewords not in $T_{n}$ and votes given to codewords not in $T_{n}$, the number of votes given by the codewords in $T_{n}$ is the same as the number of votes received by the codewords in $T_{n}$. From the voting rules we immediately see that if a codeword gives votes to another, their distance is at most 2. Consequently,

$$
\sum_{\mathbf{c} \in C \cap T_{n}} m(\mathbf{c}) \geqslant-4\left|T_{n+2} \backslash T_{n}\right|=-4(16 n+24),
$$

where $4\left|T_{n+2} \backslash T_{n}\right|$ is an upper bound (cf. Lemmas 1,2 and 3) on the total number of votes received by the codewords in $T_{n}$ from the codewords not in $T_{n}$.

On the other hand, if we consider the sum $\sum_{\mathbf{c} \in C \cap T_{n}} s(\mathbf{c})$, then every vertex $\mathbf{v} \in T_{n-1}$ with $|I(\mathbf{v})|=i$ contributes the summand $1 / i$ to $s(\mathbf{c})$ for all the $i$ codewords $\mathbf{c}$ within distance one from $\mathbf{v}$ (and these codewords $\mathbf{c}$ all belong to $T_{n}$ ). Hence

$$
\begin{aligned}
\sum_{\mathbf{c} \in C \cap T_{n}} s(\mathbf{c}) & \geqslant\left|T_{n-1}\right| \\
& =\left|T_{n}\right|-8 n .
\end{aligned}
$$

From (1) we see that $s(\mathbf{c})+m(\mathbf{c}) \leqslant 19 / 6$ for all $\mathbf{c} \in C \cap T_{n}$ and therefore

$$
\sum_{\mathbf{c} \in C \cap T_{n}}(s(\mathbf{c})+m(\mathbf{c})) \leqslant \frac{19}{6}\left|C \cap T_{n}\right| .
$$

Using (2), (3) and (4) we now see that

$$
\left|T_{n}\right|-8 n-4(16 n+24) \leqslant \sum_{\mathbf{c} \in C \cap T_{n}}(s(\mathbf{c})+m(\mathbf{c})) \leqslant \frac{19}{6}\left|C \cap T_{n}\right|,
$$

i.e.,

i.e.,

$$
\left|T_{n}\right|-(72 n+96) \leqslant \frac{19}{6}\left|C \cap T_{n}\right|,
$$

$$
\frac{\left|C \cap T_{n}\right|}{\left|T_{n}\right|} \geqslant \frac{6}{19}-\frac{6(72 n+96)}{19(2 n+1)^{2}}
$$

from which we see that the claim is true.

So we have proved:

Theorem 2. The smallest possible density of a strongly identifying code in the infinite triangular grid is $6 / 19$.

Acknowledgment: The author would like to thank the referees for many useful comments. 


\section{References}

[1] I. Charon, I. Honkala, O. Hudry and A. Lobstein, General bounds for identifying codes in some infinite regular graphs, Electron. J. Combin. 8 (2001) R39.

[2] I. Charon, O. Hudry and A. Lobstein, Identifying codes with small radius in some infinite regular graphs, Electron. J. Combin. 9 (2002) R11.

[3] T. W. Haynes, S. T. Hedetniemi and P. J. Slater, Fundamentals of Domination in Graphs (Marcel Dekker, New York, 1998).

[4] I. Honkala, An optimal robust identifying code in the triangular lattice, Ann. Comb. 8 (2004) 303-323.

[5] I. Honkala, An optimal locating-dominating set in the infinite triangular grid, Discrete Math. 306 (2006) 2670-2681.

[6] I. Honkala and T. Laihonen, On identifying codes in the triangular and square grids, SIAM J. Comput. 33 (2004) 304-312.

[7] I. Honkala, T. Laihonen and S. Ranto, On strongly identifying codes, Discr. Math. 254 (2002) 191-205.

[8] M. G. Karpovsky, K. Chakrabarty and L. B. Levitin, On a new class of codes for identifying vertices in graphs, IEEE Trans. Inform. Th. 44 (1998) 599-611.

[9] T. Laihonen, Optimal codes for strong identification, Europ. J. Combin. 23 (2002) $307-313$.

[10] T. Laihonen and S. Ranto, Families of optimal codes for strong identification, Discr. Appl. Math. 121 (2002) 203-213.

[11] A. Lobstein, Identifying, locating-dominating and discriminating codes in graphs (Internet bibliography), http://perso.telecom-paristech.fr/ lobstein/bibLOCDOMetID.html

[12] S. J. Seo and P. J. Slater, Open neighborhood locating dominating sets, submitted.

[13] P. J. Slater, Dominating and reference sets in a graph, J. Math. Phys. Sci. 22 (1988) 445-455.

[14] P. J. Slater, Fault-tolerating locating-dominating sets, Discrete Math. 249 (2002) 179-189. 